\title{
Alternativas Microquirúrgicas (Autoinjertos Nerviosos I Aloinjertos Nerviosos / Conductos Nerviosos) para la Recuperación Sensitiva Funcional de Nervio Alveolar Inferior y Nervio Lingual: Revisión de la Literatura.
}

\author{
Microsurgical Alternatives (Nerve Autografts / Nerve Allografts/ \\ Nerve Ducts) for Functional Sensory Recovery of the Lower \\ Alveolar Nerve and Lingual Nerve: Review of the Literature.
}

\author{
Edgard Riquelme-Medel ${ }^{1 *}$, Alfonso Muñoz-Roldán², Rodrigo Badilla-Monasterio ${ }^{3}$
}

\begin{abstract}
1. Cirujano Dentista, Pasante Servicio de Cirugía Oral y Traumatología Maxilofacial, Hospital Clínico Félix Bulnes Cerda, Santiago, Chile.

2. Cirujano Dentista, Docente Cátedra Anatomía Humana Universidad Mayor, Santiago, Chile. 3. Cirujano Dentista, Especialista en Cirugía Oral y Traumatología Maxilofacial, Hospital Clínico Félix Bulnes Cerda, Santiago, Chile.
\end{abstract}

* Correspondencia Autor: Dr. Edgard Riquelme Medel. | E-mail: edgard.riquelme.medel@gmail.com Trabajo recibido el 31/01/2019.

Aprobado para su publicación el 19/04/2020

\section{RESUMEN}

Objetivo: analizar y comparar las modalidades actuales de reconstrucción microquirúrgica (autoinjertos, aloinjertos y conductos nerviosos) para lograr recuperación sensitiva funcional (RSF) en reparaciones de nervio alveolar inferior (NAI) y nervio lingual (NL). Materiales y Métodos: se realizó una revisión de la literatura para identificar estudios relacionados con reconstrucciones de NAI y NL. Los estudios incluidos proporcionaron un tamaño de muestra definido, modalidad de reconstrucción microquirúrgica y tasas de RSF. Se realizó un test de proporciones para saber si existían diferencias estadísticamente significativas $(P<0,05)$ entre las modalidades de reconstrucción mencionadas. Resultados: se seleccionaron y analizaron 9 estudios que dieron como resultado una muestra de 130 reconstrucciones de NAI y 102 de NL. Entre las reconstrucciones de NAI, se encontró que los autoinjertos y aloinjertos eran superiores para lograr una RSF sobre los conductos nerviosos ( $P$ de 0,033 y 0,0397 respectivamente). Entre las reconstrucciones de NL, no se encontraron diferencias estadísticamente significativas. $\mathrm{Y}$ al comparar reconstrucciones mediante autoinjertos con aloinjertos, no hubo diferencias estadísticamente significativas para NAI y NL. Conclusiones: Las reconstrucciones mediante aloinjertos y autoinjertos tiene tasas de RSF equivalentes y mejores que los conductos nerviosos. Además, con el uso de aloinjertos, se evitan comorbilidades asociadas al sitio donante.

PALABRAS CLAVE:

Nervio alveolar inferior; Nervio lingual; Reconstrucción nerviosa.

Int. J. Inter. Dent Vol. 13(2); 84-87, 2020.

\section{ABSTRACT}

Objective: to analyze and compare the current modalities of microsurgical reconstruction (autografts, allografts, and nerve ducts) to achieve functional sensory recovery (RSF) in repairs of the inferior alveolar nerve (NAI) and lingual nerve (NL). Materials and Methods: a literature review was performed to identify studies related to $\mathrm{NAI}$ and $\mathrm{NL}$ reconstructions. The studies included provided a defined sample size, microsurgical reconstruction modality, and RSF rates. A test of proportions was performed to find out if there were statistically significant differences $(P<0.05)$ between the mentioned reconstruction modalities. Results: 9 studies were selected and analyzed that resulted in a sample of 130 reconstructions of NAI and 102 of NL. Among the NAI reconstructions, autografts and allografts were found to be better in achieving an RSF than the nerve ducts ( $P$ of 0.033 and 0.0397 respectively). Among the NL reconstructions, no statistically significant differences were found. And when comparing reconstructions using autografts with allografts, there were no statistically significant differences for NAI and NL. Conclusions: Reconstructions using allografts and autografts have equivalent and better RSF rates than nerve conduits. Furthermore, with the use of allografts, comorbidities associated with the donor site are avoided. KEY WORDS:

Inferior alveolar nerve; Lingual nerve; Nerve reconstruction.

Int. J. Inter. Dent Vol. 13(2); 84-87, 2020. 


\section{INTRODUCCIÓN}

Las lesiones del nervio trigémino tienen un impacto negativo en la calidad de vida de los pacientes. Pueden causar entumecimiento, parestesia, ardor, alteración del gusto, habla, masticación, retención de saliva y/o dolor neuropático dentro de su distribución anatómica ${ }^{(1,2)}$. Los ramos del nervio trigémino que con mayor frecuencia se lesionan, debido a su ubicación y distribución anatómica variable, son el nervio alveolar inferior (NAI) y nervio lingual (NL). Una de las causas más comunes de lesión iatrogénica del NAl y NL son las exodoncias de terceros molares; el $4.4 \%$ de los pacientes intervenidos quirúrgicamente desarrollan parestesia temporal y el $1 \%$ desarrollan parestesia permanente ${ }^{(3)}$. Otras causas comunes incluyen implantes dentales, procedimientos endodónticos, traumatismos, extracciones de dientes, resección mandibular debido a tumores locales, osteorradionecrosis y osteomielitis ${ }^{(2,4)}$.

Existen varias opciones reconstructivas de un nervio periférico. Una de las técnicas más antiguas y utilizadas para la reconstrucción primaria es la neurorrafia directa; la cual está indicada cuando el nervio afectado puede ser suturado sin tensión entre los segmentos nerviosos. La regeneración nerviosa puede verse afectada negativamente por la tensión entre los cabos del nervio dañado, comprometiendo el flujo sanguíneo nutritivo intrafascicular ${ }^{(5,6)}$

Si no se puede realizar una neurorrafia directa libre de tensión, existen tres modalidades de reparación sin tensión mediante las cuales se puede reconstruir la brecha nerviosa. La primera modalidad es el uso de autoinjertos nerviosos. Los nervios autólogos más comúnmente utilizados para las lesiones del nervio trigémino son los nervios auriculares y surales mayores por sus características anatómicas similares al NAI y NL ${ }^{(7,8)}$. El uso de autoinjertos para la reconstrucción microquirúrgica de lesiones del nervio trigémino ha sido bien documentado, con resultados positivos en estudios recientes que van desde 87.3 a $100 \%{ }^{(9,10)}$. Sin embargo, esta técnica necesita un procedimiento quirúrgico adicional para la obtención del tejido nervioso del paciente; por lo que pueden surgir complicaciones debido a las comorbilidades del sitio donante, que incluyen problemas de cicatrización de heridas, formación de neuromas dolorosos o pérdida permanente de sensibilidad ${ }^{(7,11)}$

La segunda modalidad son los aloinjertos nerviosos procesados que corresponden a un andamio de matriz extracelular (laminina, fibronectina y glicosaminoglicanos) creado a partir de tejido nervioso periférico humano cadavérico. Una vez recuperado del donante, el aloinjerto se limpia para eliminar las células y los desechos celulares, se trata con enzimas para suprimir los inhibidores que se producen naturalmente en la regeneración axonal y se esteriliza con radiación gamma. Descelularizar y esterilizar el aloinjerto reduce significativamente el riesgo de rechazo inmune y por lo tanto, elimina la necesidad de terapia inmunosupresora ${ }^{(12,13)}$. Después de procesamiento se almacenan congelados a menos de $40{ }^{\circ} \mathrm{C}$. Antes de la implantación, los aloinjertos se descongelan completamente en solución salina estéril o de Ringer Lactato para luego ser instalados mediante la misma técnica utilizada para implantar un nervio autólogo. Por otro lado, el uso de estos aloinjertos nerviosos evita las complicaciones propias del sitio donante y sus comorbilidades asociadas con la extracción de los autoinjertos. Además, los aloinjertos han demostrado ser clínicamente eficaces y seguros para las discontinuidades nerviosas periféricas de 5 a $50 \mathrm{~mm}^{(14)}$.

La tercera modalidad son los conductos nerviosos. Corresponden a dispositivos que se utilizan como armazón a través del cual se produce el proceso de regeneración nerviosa. La regeneración a través de estos conductos se logra predominantemente a través de un cable de fibrina formado entre los muñones nerviosos proximal y distal. Existen tres generaciones. Los de primera generación son sintéticos y están hechos de materiales no reabsorbibles, como la silicona. Los de segunda generación están compuestos de materiales reabsorbibles como el colágeno Tipo I, ácido poliglicólico o submucosa intestinal de porcino. Los de tercera generación están actualmente en desarrollo e intentan incorporar células madres o de Schwann, proteínas de la matriz extracelular, entre otras ${ }^{(15,16)}$. Sin embargo, los datos clínicos actuales apoyan el uso de estos dispositivos para la reconstrucción de brechas nerviosas no mayores a $6 \mathrm{~mm}^{(17)}$. Además, se ha encontrado que los conductos de segunda generación son efectivos para promover la regeneración nerviosa después de la neurorrafia directa, logrando obtener una recuperación sensitiva superior a S3 en la escala del Consejo de Investigación Médica $(\mathrm{CIM})^{(18)}$. La escala va de S0 a S4 y se considera una recuperación sensitiva funcional (RSF) en una puntuación de S3 o superior (Tabla 1) (18,19). $^{(2)}$

El objetivo de esta revisión es analizar los estudios más recientes relacionados con reparaciones microquirúrgicas de NAl y NL. Además, determinar qué modalidad de tratamiento es más eficaz según la literatura disponible para la reconstrucción de brecha nerviosa de NAl y NL.
Tabla 1. Clasificación de la recuperación sensitiva (escala del CIM).

\begin{tabular}{|l|l|}
\hline \multicolumn{1}{|c|}{$\begin{array}{c}\text { Grado } \\
\text { (Etapa) }\end{array}$} & \multicolumn{1}{|c|}{ Recuperación de la Sensibilidad } \\
\hline S0 & Sin recuperación \\
\hline S1 & Recuperación del dolor cutáneo profundo \\
\hline S1+ & Recuperación del algún dolor superficial \\
\hline S2 & Retorno de algún dolor superficial y sensación táctil \\
\hline S2+ & S2 con exceso de respuesta (sobre-respuesta) \\
\hline S3 & $\begin{array}{l}\text { Retorno de algún dolor superficial y sensación táctil sin sobre- } \\
\text { respuesta; Discriminación de dos puntos > 15 mm }\end{array}$ \\
\hline S3+ & $\begin{array}{l}\text { S3 con buena localización del estímulo; Descriminación de dos } \\
\text { puntos = 7-15 mm }\end{array}$ \\
\hline S4 & Recuperación completa; S3; Descriminación de dos puntos $=2-6 \mathrm{~mm}$ \\
\hline CIM: Consejo de Investigación Médica.
\end{tabular}

\section{MATERIALES Y MÉTODOS}

Se realizó una revisión de la literatura relacionada con reconstrucciones de NAl y NL para identificar estudios que se centraran en la recuperación sensitiva después de la reparación microquirúrgica de estos nervios. La búsqueda se llevó a cabo en las bases de datos PubMed, EBSCO y Epistemonikos utilizando las palabras claves "inferior alveolar nerve", "lingual nerve" y "nerve reconstruction" relacionadas entre sí con términos booleanos. Se utilizaron como criterios de inclusión: artículos relativos a la pregunta de investigación, estudios in vivo, de no más de 10 años de publicación, en idioma inglés o español y free-full text. De los estudios encontrados, se filtraron por título, resumen y lectura de texto completo, excluyendo aquellos trabajos que hacían referencia a otras ramas del nervio trigémino, pacientes ya tratados anteriormente con algún tipo de técnica regeneración nerviosa, estudios en animales y estudios que directamente escapaban al área de cabeza y cuello. Los parámetros de interés que se incluyeron al momento de realizar la lectura crítica fueron: tipos de estudios (retrospectivo, prospectivo y comparativo), la técnica quirúrgica, material utilizado para la reconstrucción nerviosa con una muestra $(n)$ bien definida y calidad de la recuperación sensitiva según escala CIM. Consideramos estudios cuya recuperación de la sensibilidad tuvieran una puntuación de S3 o superior en la escala CIM (RSF). Se realizó un Test de Proporciones para saber si existían diferencias estadísticamente significativas $(P<0,05)$ entre las modalidades de reconstrucción para brecha nerviosa (autoinjertos nerviosos / aloinjertos nerviosos / conductos nerviosos). Por último, se consideró la existencia de consentimiento informado, la aprobación de un comité de ética y la ausencia de conflictos de interés.

\section{RESULTADOS}

Se encontraron durante la búsqueda un total de 56 estudios de los

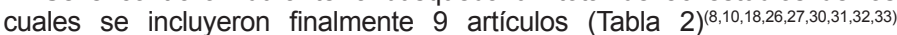
según los criterios de inclusión y exclusión. Se obtuvieron un total de 232 reconstrucciones microquirúrgicas, de las cuales 130 (56\%) correspondían a reconstrucciones de NAl y $102(44 \%)$ a reconstrucciones de NL.

De las reconstrucciones de NAl, 18 fueron reparaciones primarias aisladas y 112 necesitaron el uso de un material para el cierre de brecha sin tensión entre los segmentos nerviosos (autoinjertos nerviosos / aloinjertos nerviosos / conductos nerviosos). De estas reconstrucciones de brecha nerviosa, 71 fueron mediante autoinjertos, 34 mediante aloinjertos y 7 reconstrucciones mediante conductos.

De las reconstrucciones de NL, 20 fueron reparaciones primarias aisladas, 43 fueron reparaciones primarias complementadas con el uso de conductos y 39 necesitaron de un material para el cierre brecha sin tensión entre los segmentos nerviosos (autoinjertos nerviosos / aloinjertos nerviosos / conductos nerviosos). De estas reconstrucciones de brecha nerviosa, 24 fueron autoinjertos, 12 aloinjertos y 3 reconstrucciones mediante conductos.

De las reconstrucciones de NAI, lograron RSF: 16 (89\%) de 18 reparaciones primarias, $62(87 \%)$ de 71 reconstrucciones mediante autoinjertos, $30(88 \%)$ de 34 reconstrucciones mediante aloinjertos y 3 $(43 \%)$ de 7 reconstrucciones mediante conductos. De las reconstrucciones de NL, lograron RSF: 17 (85\%) de 20 reparaciones primarias, $43(100 \%)$ de 43 reparaciones primarias complementadas con el uso de conductos, $21(88 \%)$ de 24 reconstrucciones mediante autoinjertos, $12(100 \%)$ de 12 reconstrucciones mediante aloinjertos y $3(100 \%)$ de 3 reconstrucciones mediante conductos (Tabla 3 ). Las brechas nerviosas oscilaron entre 10 y 70 
Tabla 2. Lista de estudios seleccionados y su descripción en cuanto al tipo de nervio, tipo de reconstrucción y sus resultados.

\begin{tabular}{|c|c|c|c|c|c|c|}
\hline Estudios & An̂o & Reparaciones Nerviosas (n) & Tipo de Reconstrucción & $\begin{array}{l}\text { Longitud de la } \\
\text { Brecha }\end{array}$ & $\begin{array}{l}\text { Recuperación } \\
\text { Sensitiva } \\
\text { Funcional }\end{array}$ & $\begin{array}{c}\text { Porcentaje de } \\
\text { Recuperacion } \\
\text { Sensitiva Funcional }\end{array}$ \\
\hline Shanti \& Ziccardi ${ }^{|3|}$ & 2011 & $1 \mathrm{NAl}$ & Aloinjertos & Desconoclda & 1 NAI & $100 \%$ \\
\hline Bagheri et al ${ }^{(10)}$ & 2012 & $89 \mathrm{NAl}$ & $\begin{array}{c}18 \text { primarias } \\
71 \text { autoinjertos }\end{array}$ & $>20 \mathrm{~mm}$ & $\begin{array}{c}16 \text { primarias } \\
62 \text { autoinjertos }\end{array}$ & $87,6 \%$ \\
\hline Miloro et al. ${ }^{(8)}$ & 2015 & $47 \mathrm{NL}$ & $\begin{array}{c}19 \text { primarias } \\
4 \text { aloinjertos } \\
24 \text { autoinjertos }\end{array}$ & $\begin{array}{c}10-30 \mathrm{~mm} \\
\text { (promedio } 14.3 \mathrm{~mm} \text { ) }\end{array}$ & $\begin{array}{c}16 \text { primarias } \\
4 \text { aloinjertos } \\
21 \text { autoinjertos }\end{array}$ & $87,2 \%$ \\
\hline Salomon et al. ${ }^{(32)}$ & 2016 & $7 \mathrm{NAl}$ & Aloinjertos & $\begin{array}{c}50-70 \mathrm{~mm} \\
\text { (promedio } 67.1 \mathrm{~mm} \text { ) }\end{array}$ & 6 NAl & $85,7 \%$ \\
\hline Theberge \& Ziccardi $|2 \pi|$ & 2016 & $1 \mathrm{NL}$ & Primaria & $0 \mathrm{~mm}$ & $1 \mathrm{NL}$ & $100 \%$ \\
\hline Seo et al. ${ }^{(33)}$ & 2016 & $\begin{array}{l}7 \mathrm{NAl} \\
3 \mathrm{NL}\end{array}$ & $\begin{array}{l}\text { Conductos de tubos } \\
\text { (PGA-c Tube) }\end{array}$ & Desconocida & $\begin{array}{l}3 \mathrm{NAl} \\
3 \mathrm{NL}\end{array}$ & $60 \%$ \\
\hline Wilson et al. ${ }^{(18)}$ & 2016 & $43 \mathrm{NL}$ & $\begin{array}{c}\text { Primarias + conductos } \\
\text { (NeuraGen@/AxoGuard@) }\end{array}$ & Sin brecha & $43 \mathrm{NL}$ & $100 \%$ \\
\hline Yampolsky et al. ${ }^{(30)}$ & 2017 & $\begin{array}{l}8 \mathrm{NAl} \\
8 \mathrm{NL}\end{array}$ & Aloinjertos & $<20 \mathrm{~mm}$ & $\begin{array}{l}7 \mathrm{NAl} \\
8 \mathrm{NL}\end{array}$ & $93,8 \%$ \\
\hline Zuñiga et al. 221$)$ & 2017 & $18 \mathrm{NAl}$ & $\begin{array}{l}18 \text { aloinjertos } \\
\text { ( } 2 \text { controles) }\end{array}$ & $\begin{array}{c}45-70 \mathrm{~mm} \\
\text { (promedio } 62.7 \mathrm{~mm} \text { ) }\end{array}$ & 16 aloinjertos & $88,9 \%$ \\
\hline
\end{tabular}

AxoGuarde: conducto de tubo en base a submucosa de intestino delgado de porcino, NeuraGene: conducto de tubo en base a colégeno tipo l, PGA-c Tube: conducto de tubo en base a ácido poliglícólico.

$\mathrm{mm}$. Sin embargo, la brecha promedio por subgrupo no se pudo determinar debido a la escasez de datos. El tiempo desde la alteración neurosensitiva inicial hasta la reparación microquirúrgica, la etiología de la lesión nerviosa, la demografía y las comorbilidades de los pacientes tampoco pudo ser subgrupado y analizado adecuadamente debido a la heterogeneidad y la falta de disponibilidad de los datos.

A los resultados obtenidos de porcentajes de RSF tanto para NL como NAI, se les aplicó un Test de Proporciones para comparar las diferentes modalidades de reconstrucción para brecha nerviosa (autoinjertos

Tabla 3. Tasas de recuperación sensitiva funcional (RSF) de NAI y NL según modalidad de reconstrucción nerviosa.

\begin{tabular}{|l|c|c|c|c|}
\hline \multicolumn{1}{|c|}{$\begin{array}{c}\text { Tipo de } \\
\text { Reconstrucción }\end{array}$} & $\begin{array}{c}\text { NAl: } \\
\text { Relación } \\
\text { RSFTrtalal }\end{array}$ & $\begin{array}{c}\text { NAl: } \\
\% \text { de Éxito }\end{array}$ & $\begin{array}{c}\text { NL: } \\
\text { Relación } \\
\text { RSF/Total }\end{array}$ & $\begin{array}{c}\text { NL: } \\
\% \text { de Éxito }\end{array}$ \\
\hline Primaria & $16 / 18$ & $88,9 \%$ & $17 / 20$ & $85 \%$ \\
\hline Primaria + conductos & - & - & $43 / 43$ & $100 \%$ \\
\hline Autoinjertos & $62 / 71$ & $87,3 \%$ & $21 / 24$ & $87,5 \%$ \\
\hline Aloinjertos & $30 / 34$ & $88,2 \%$ & $12 / 12$ & $100 \%$ \\
\hline Conductos & $3 / 7$ & $42,6 \%$ & $3 / 3$ & $100 \%$ \\
\hline
\end{tabular}

nerviosos / aloinjertos nerviosos / conductos nerviosos). Se determinó que, para el NAI, existen diferencias estadísticamente significativas entre los tratamientos mediante autoinjertos/aloinjertos y los tratamientos en base a conductos en cuanto a la RSF con valores de $\mathrm{P}$ de 0,033 y 0,0397 respectivamente. Entre las reconstrucciones de la brecha nerviosa para el NL, no se encontraron diferencias estadísticamente significativas entre las tres modalidades de tratamientos. Al comparar los resultados de la reconstrucción mediante autoinjertos con aloinjertos, no hubo diferencias estadísticamente significativas en la RSF para NAI y NL (Tabla 4). No se realizaron test estadísticos para la modalidad de tratamiento primario y primario complementado con conductos, ya que no son tratamientos indicados cuando existen brechas en injurias nerviosas.

\section{DISCUSIÓN}

A pesar de que actualmente hay mejoras en las técnicas quirúrgicas y del uso de planificación virtual en cirugía maxilofacial, siguen ocurriendo
Tabla 4. Resultados del test de proporciones entre las diferentes modalidades de reconstrucción para brecha nerviosa.

\begin{tabular}{|l|c|}
\hline & $P$ \\
\hline $\begin{array}{l}\text { NAl Autoinjertos vs } \\
\text { Aloinjertos }\end{array}$ & 0,9023 \\
\hline $\begin{array}{l}\text { NAl Autoinjertos vs } \\
\text { Conductos }\end{array}$ & $\mathbf{0 , 0 3 3}$ \\
\hline $\begin{array}{l}\text { NAl Aloinjertos vs } \\
\text { Conductos }\end{array}$ & $\mathbf{0 , 0 3 9 7}$ \\
\hline $\begin{array}{l}\text { NL Autoinjertos vs } \\
\text { Aloinjertos }\end{array}$ & 0,2018 \\
\hline $\begin{array}{l}\text { NL Autoinjertos vs } \\
\text { Conductos }\end{array}$ & 0,5164 \\
\hline $\begin{array}{l}\text { NL Aloinjertos vs } \\
\text { Conductos }\end{array}$ & 1 \\
\hline
\end{tabular}

NAl: Nervio Alveolar Inferior, NL: Nervio Lingual.

lesiones de los NAI y NL ${ }^{(10,20,21)}$. Debido a la naturaleza de las lesiones de estos nervios es importante considerar la reconstrucción microquirúrgica para optimizar la RSF y la calidad de vida del paciente(1). Por lo tanto, es necesario evaluar las actuales modalidades de reparación nerviosa para poder guiar a los pacientes sobre sus mejores opciones de tratamiento. Además, es fundamental conocer las ventajas y desventajas de las diferentes modalidades de reconstrucción para ofrecer a nuestros pacientes la alternativa más adecuada.

Según los resultados obtenidos para las reparaciones primarias de NAI y NL, las tasas de RSF fueron del $89 \%$ y del $85 \%$ respectivamente. De esta manera, no existen diferencias estadísticamente significativas entre los dos nervios para las reconstrucciones primarias, pero puede haber diferencias con un tamaño de muestra mayor. Esto debido a que el NAI se encuentra relativamente inmóvil dentro de un canal óseo, mientras que el NL está libre y puede movilizarse más para obtener una reparación primaria sin tensión. Por lo tanto, es importante tener en consideración este factor anatómico en caso de resultados discrepantes a los obtenidos en esta revisión, ya que cualquier diferencia en la recuperación sensitiva entre ambos nervios puede estar relacionada con un aumento de la tensión debido a una incapacidad para movilizar adecuadamente el NAI y asegurar una reparación libre de tensión.

Cuando la reparación nerviosa sin tensión no es posible, la brecha se debe reconstruir con autoinjertos, aloinjertos o conductos nerviosos ${ }^{(16,20,22)}$. 
En los estudios evaluados, se encontró que los tratamientos en base a conductos eran menos efectivos que los tratamientos con autoinjertos y aloinjertos en reconstrucciones de NAI. Pero para reconstrucciones de $\mathrm{NL}$ no se encontraron diferencias estadísticamente significativas entre las tres modalidades de tratamiento. Cabe mencionar que la muestra de tratamientos en base a conductos para NAI y NL era escasa porque es un tratamiento que actualmente no se realiza con tanta frecuencia como el uso de aloinjertos. En estudios recientes de otras reconstrucciones nerviosas se encontró que los conductos nerviosos son exitosos para lograr una RSF en brechas cortas. Safa y Buncke encontraron que, en brechas de menos de $6 \mathrm{~mm}$, los conductos lograron una RSF de manera consistente. Al contrario, En brechas mayores de $6 \mathrm{~mm}$, la tasa de RSF disminuyó rápidamente y los resultados fueron significativamente peores en brechas más largas ${ }^{(23)}$. Se requiere un tamaño de muestra mayor para evaluar y respaldar completamente el uso limitado de conductos en pequeñas brechas nerviosas.

En el mismo estudio de Safa y Buncke ${ }^{(23)}$, se encontró que los aloinjertos lograban una RSF en brechas nerviosas de hasta $70 \mathrm{~mm}$, lo que concuerda con los hallazgos encontrados en esta revisión. Se cree que esta disparidad entre ambas modalidades de reconstrucción nerviosa se debe a las diferencias estructurales entre ellas. El aloinjerto conserva la arquitectura del nervio y el microambiente de la matriz extracelular, que a su vez promueve un crecimiento guiado natural de las fibras nerviosas: mientras que los conductos no tienen estas características. Además, en este estudio se encontró que el aloinjerto tuvo resultados favorables similares al autoinjerto, lo que también concuerda con los hallazgos encontrados en esta revisión. Sin embargo, una desventaja importante de los autoinjertos es que además de requerir tiempo adicional de extracción quirúrgica, se han asociado con complicaciones en el sitio del donante, que incluyen la necesidad de incisiones adicionales, posibles problemas de curación de heridas, formación de neuromas dolorosos y pérdida sensorial en el sitio del donante ${ }^{(24,25)}$. Por lo tanto, se debe preferir el uso de aloinjertos ya que evitaría cualquier posible complicación en el sitio donante y se ha demostrado que tiene resultados de RSF similares a los autoinjertos.

Aunque los conductos pueden tener una indicación limitada en la reconstrucción para brecha nerviosa, varios estudios respaldan su uso como complementos de las reparaciones primarias y de las reconstrucciones de brecha mediante aloinjertos ${ }^{(18,21,26,27)}$. Funcionan como una barrera física entre el nervio reparado y el entorno externo, que protege y contiene las proteínas de la matriz extracelular necesarias para una curación óptima en el sitio de reparación, así como para prevenir el crecimiento excesivo y el escape axonal| ${ }^{28,29,30,31,32)}$. Wilson et al., realizaron un estudio comparando envolturas de conducto de colágeno de tipo I con envolturas de submucosa de intestino delgado porcino en reparaciones primarias de NL. Encontraron que no había diferencias en las tasas de RSF para ninguno de los grupos y que los 43 pacientes incluidos en el estudio tenían una puntuación igual o superior a S3 en la escala $\mathrm{CIM}^{(18)}$. Si bien el tamaño de la muestra del estudio no fue lo suficientemente grande como para demostrar diferencias estadísticamente significativas entre las reparaciones envueltas sin conector y las reparaciones envueltas con conector, creemos que este es un complemento útil para optimizar la recuperación sensitiva ${ }^{(28,29,33)}$.

\section{CONCLUSIONES}

La reparación microquirúrgica de los NAI y NL permite una recuperación sensitiva eficaz y mejora la calidad de vida de los pacientes.

La mejor alternativa de tratamiento para las reconstrucciones de NAI y NL es la reparación primaria libre de tensión; de no existir esta posibilidad, los aloinjertos tienen un mejor comportamiento que los autoinjertos, ya que las tasas de RSF entre ambas modalidades de tratamientos son equivalentes, pero con el uso de los aloinjertos se evitan las comorbilidades asociadas al sitio donante.

Hacen falta estudios experimentales de reconstrucciones nerviosas mediante conductos para establecer una casuística certera con respecto a este tipo de tratamiento.

\section{CONFLICTO DE INTERESES}

Los autores declaran que no tienen ningún conflicto de intereses.

\section{FUENTES DE FINANCIAMIENTO}

Este trabajo no cuenta con financiamiento alguno.

\section{Bibliografía}

1. Smith JG, Elias LA, Yilmaz Z, Barker S, Shah K, Shah S, et al. The psychosocial and affective burden of posttraumatic neuropathy following injuries to the trigeminal nerve. $J$ Orofac Pain. 2013;27:293-303

2. Zuniga JR, Yates DM. Factors determining outcome after trigeminal nerve surgery for neuropathic pain. J Oral Maxillofac Surg. 2016;74:1323-1329.

3. Friedman JW. The prophylactic extraction of third molars: a public health hazard. Am J Public Health. 2007;97:1554

4. McClary AC, West RB, McClary AC, Pollack JR, Fischbein NJ, Holsinger CF, et al. Ameloblastoma: a clinical review and trends in management. Eur Arch Otorhinolaryngol. 2016;273:1649-1661

5. Lundborg G, Rydevik B. Effects of stretching the tibial nerve of the rabbit. A preliminary study of the intraneural circulation and the barrier function of the perineurium. J Bone Joint Surg Br. 1973;55B:390.

6. Clark WL, Trumble TE, Swiontkowski MF, Tencer AF. Nerve tension and blood flow in a rat model of immediate and delays repairs. J Hand Surg Am. 1992;17:677.

7. Taraquois R, Joly A, Sallot A, Kün Darbois JD, Laure B, Paré A. Inferior alveolar nerve reconstruction after segmental resection of the mandible. Rev Stomatol Chir Maxillofac Chir Orale. 2016;117:438-441.

8. Miloro M, Ruckman P 3rd, Kolokythas A. Lingual nerve repair: to graft or not to graft? J Oral Maxillofac Surg. 2015;73:1844-1850.

9. Miloro M, Stoner JA. Subjective outcomes following sural nerve harvest. J Oral Maxillofac Surg. 2005;63:1150.

10. Bagheri SC, Meyer RA, Cho SH, Thoppay J, Khan HA, Steed MB. Microsurgical repair of the inferior alveolar nerve: Success rate and factors that adversely affect outcomes. J Oral Maxillofac Surg. 2012;70:1978.

11. Martins RS, Barbosa RA, Siqueira MG, Soares MS, Heise CO, Foroni L, et al. Morbidity following sural nerve harvesting: a prospective study. Clin Neurol Neurosurg. 2012;114:1149-1152.

12. Wolford LM, Rodrigues DB. Autogenous grafts/allografts/conduits for bridging peripheral trigeminal nerve gaps. Atlas Oral Maxillofac Surg Clin North Am. 2011;19:91107.

13. Whitlock EL, Tuffaha SH, Luciano JP, Yan Y, Hunter DA, Magill CK, et al. Processed allografts and type I collagen conduits for repair of peripheral nerve gaps. Muscle Nerve. 2009;39:787-799.

14. Cho MS, Rinker BD, Weber RV, Chao JD, Ingari JV, Brooks D, et al. Functional outcome following nerve repair in the upper extremity using processed nerve allograft. J Hand Surg. 2012;37A:2340.

15. Gaudin R, Knipfer C, Henningsen A, Smeets R, Heiland M, Hadlock T. Approaches to peripheral nerve repair: generations of biomaterial conduits yielding to replacing autologous nerve grafts in craniomaxillofacial surgery. Biomed Res Int. 2016;2016:3856262.

16. Lundborg G. A 25-year perspective of peripheral nerve surgery: evolving neuroscientific concepts and clinical significance. J Hand Surg Am. 2000:25:391-414.
17. Lohmeyer JA, Kern Y, Schmauss D, Paprottka F, Stang F, Siemers F, et al Prospective clinical study on digital nerve repair with collagen nerve conduits and review of literature. J Reconstr Microsurg. 2014;30:227-234

18. Wilson MT, Chuang SK, Ziccardi VB. Lingual nerve microsurgery outcomes using 2 different conduits: a retrospective cohort study. J Oral Maxillofac Surg. 2016;75:609-615. 19. Wang Y, Sunitha M, Chung KC. How to measure outcomes of peripheral nerve surgery. Hand Clin. 2013;29:349-361.

20. Zuniga JR. Sensory outcomes after reconstruction of lingual and inferior alveolar nerve discontinuities using processed nerve allograft - a case series. J Oral Maxillofac Surg. 2014;73:734-744

21. Miloro M, Markiewicz MR. Virtual surgical planning for inferior alveolar nerve reconstruction. J Oral Maxillofac Surg. 2017;75:2442-2448.

22. Brooks DN, Weber RV, Chao JD, Rinker BD, Zoldos J, Robichaux MR, et al. Processed nerve allografts for peripheral nerve reconstruction: a multicenter study of utilization and outcomes in sensory, mixed, and motor nerve reconstruction. Microsurgery. 2012;32:1-14

23. Safa B, Buncke G. Autograft substitutes: conduits and processed nerve allografts. Hand Clin. 2016;32:127-140.

24. IJpma FF, Nicolai JP, Meek MF. Sural nerve donor-site morbidity: thirty-four years of follow-up. Ann Plast Surg. 2006;57:391-395

25. Meek MF, Coert JH, Robinson PH. Poor results after nerve grafting in the upper extremity: quo vadis? Microsurgery. 2005;25:396-402.

26. Theberge NP, Ziccardi VB. Use of fibrin glue as an adjunct in the repair of lingual nerve injury: case report. J Oral Maxillofac Surg. 2016;74:1899.e1-1899.e4.

27. Zuniga JR, Williams F, Petrisor D. A case-and-control, multisite, positive controlled prospective study of the safety and effectiveness of immediate inferior alveolar nerve processed nerve allograft reconstruction with ablation of the mandible for benign pathology. J Oral Maxillofac Surg. 2017;75:1-13.

28. Ducic I, Safa B, DeVinney E. Refinements of nerve repair with connector-assisted coaptation. Microsurgery. 2017;37:256-263.

29. Lee JY, Parisi TJ, Friedrich PF, Bishop AT, Shin AY. Does the addition of a nerve wrap to a motor nerve repair affect motor outcomes? Microsurgery. 2014;34:562-567.

30. Yampolsky A, Zicarrdi V, Chuang SK. Efficacy of a cellular nerve allografts in trigeminal nerve reconstruction. J Oral Maxillofac Surg. 2017;75:2230-2234.

31. Shanti RM, Ziccardi VB. Use of decellularized nerve allograft for inferior alveolar nerve reconstruction: a case report. J Oral Maxillofac Surg. 2011;69:550-553.

32. Salomon D, Miloro M, Kolokythas A. Outcomes of immediate allograft reconstruction of long-span defects of the inferior alveolar nerve. J Oral Maxillofac Surg. 2016;74:25072514 .

33. Seo K, Terumitsu M, Inada Y, Nakamura T, Shigeno K, Tanaka Y. prognosis after surgical treatment of trigeminal neuropathy with a PGA-c Tube: Report of 10 cases. Pain Med. 2016;17(12):2360-2368. 